\title{
Regularized NLMS Adaptive Algorithm for Noise Cancellation in Speech Signals
}

\author{
G.Amjad Khan ${ }^{1}$, Dr. K E Sreenivasa Murthy ${ }^{2}$ \\ ${ }^{I}$ (Assistant Professor, ECE Department,G.Pulla Reddy Engg College(Autonomous),Kurnool,A.P. India- 518007 \\ ,Affiliated to JNTUA \\ ${ }_{2}^{2}$ (Principal,Sri Sai Institute of Technology,Raichoti,KadapaDtA.P, India-516269,Affiliated to JNTUA)
}

\begin{abstract}
Adaptive noise cancellation (ANC) is widely used to reduce noise from a noisy speech signal .However the Least Mean Square algorithm and its variants, such as the Least Mean Square Algorithm (LMS), normalized (N) - LMS algorithm and the constrained stability (CS)-LMS algorithms do not perform well in ANC since the desired speech signal has a bad effect on the convergence rate and steady state misadjustments of these algorithms. Thus, we propose a new adaptive algorithm that further relaxes the constrained in the CSLMS algorithm. The new algorithm attempts to minimize the estimation error of the a posteriori error and the estimation is obtained using the concept of regularization. The analysis and simulation results show that the proposed new algorithm outperforms the LMS, NLMS and CS -LMS algorithm.
\end{abstract}

Index Terms:Adaptive noise canceller, least-mean square (LMS) algorithm, Normalised LMS algorithm speech enhancement, stability constraint

\section{Introduction}

The widely used least-mean-square (LMS) algorithm has been successfully applied to many filtering applications, including noise cancellation application, signal modelling, equalization, control, echo cancellation, biomedicine, or beam forming [1]. The typical noise cancellation scheme is shown in Figure.1. The adaptive noise canceller for filtering speech signals has two input signals .The primary input signal $d(n)$ is the desired speech signal corrupted by noise signal $n(n)$ and the reference input signal $x(n)$ correlated with $n(n)$.Due to this correlation, the adaptive canceller can reduce the noise from the primary signal and obtain the output error signal $e(n)$ equal to the speech signal $x(n)$. The correlation between the additive noise that corrupts the clean speech (primary signal) and the random noise in the reference input (adaptive filter input) is necessary to adaptively cancel the noise of the primary signal.

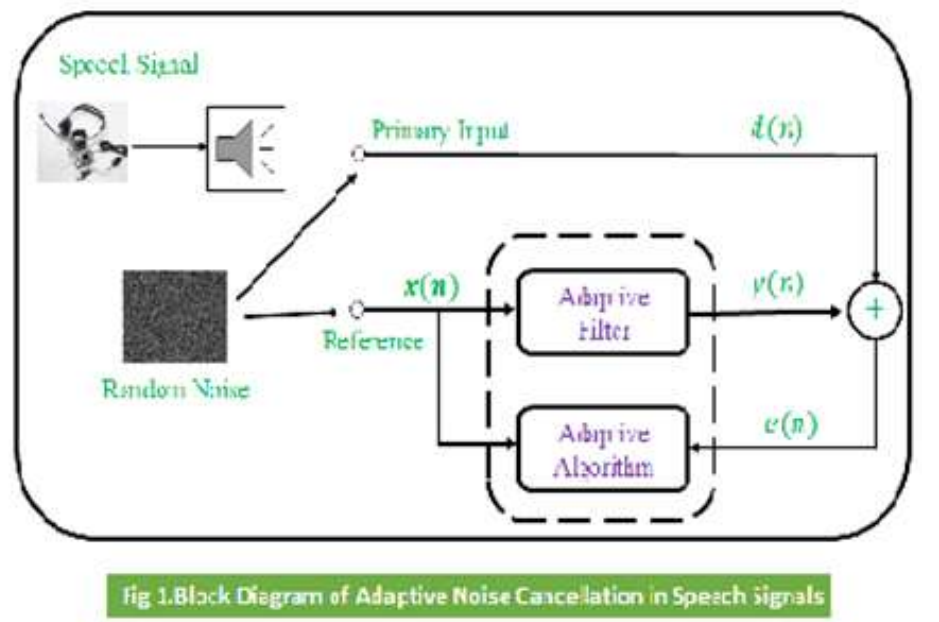

Input Signal:A speech signal can be considered as a special case of the correlated input data. We will use it in various experiments to show the ability of the algorithm to deal with non-stationary signals. However, Speech signals usually possess both, noise-like parts, which are more or less uncorrelated, and periodic parts during which the signal is highly correlated. For the testing we conducted several recordings in various acoustic environments. 


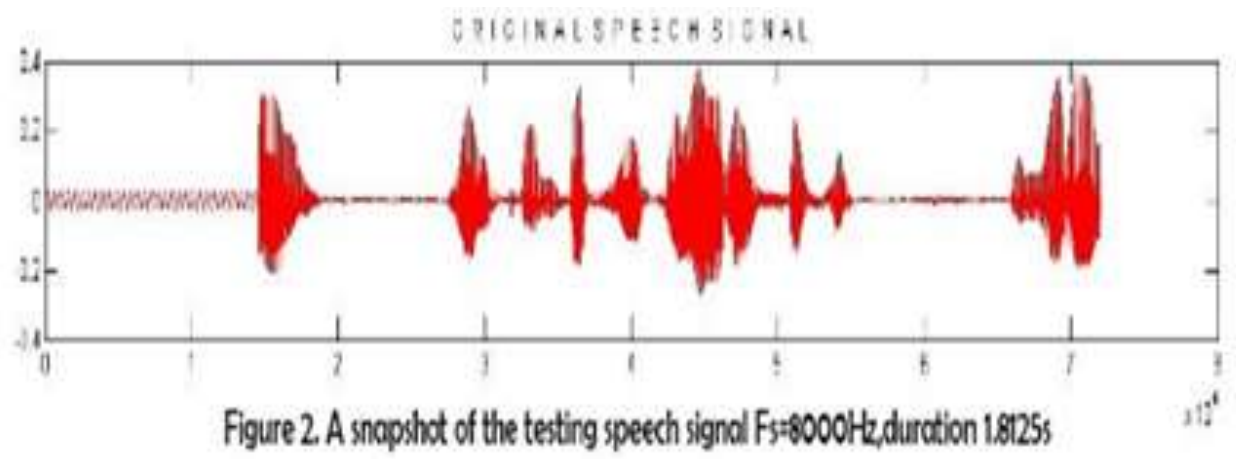

\section{Adaptive Algorithms}

2.1 Reference Algorithm 1- The adjustable weights are typically determined by the LMS algorithm [1] because of its simplicity, ease of implementation and low computational complexity. The weight update equation for the adaptive noise canceller (ANC) is

$$
e(n)=d(n)-W^{T}(n) x(n)
$$

$w(n+1)=w(n)+\mu e^{*}(n) x(n)$

Where $\mu$ is the step size parameter, $e^{*}(n)$ denotes the complex conjugate of the error signal $e(n)$ and $\left.\left.\boldsymbol{x}(\boldsymbol{n})=[x(n) x(n-1) \ldots \ldots x(N-L+1)]^{T}\right)\right]$ is the data vector containing $L$ samples of the input signal.

Major Drawback:

Advantages:

Less computational complexity.

Good convergence rate.

Disadvantages:

Drawback of LMS Algorithm:

Smaller value of step size leads to a small steady state Misadjustment (SSM) but a slower convergence rate.

Larger value of step size gives faster convergence rate but a large steady state misadjustment

\subsection{Reference Algorithm 2- The Normalised Least Mean Square Algorithm (NLMS):}

The NLMS is definitely the most popular algorithm which has been used for the past 40 years. This algorithm conceptually stems from the Least Mean Squares (LMS), which has been developed by Widrow and Hoff. The LMS algorithm is a stochastic gradient method that updates iteratively the coefficients of its own adaptive filter (tap weights) in a direction of the gradient vector. This vector is calculated as a partial derivative of the Mean-Square Error (MSE) function with respect to the tap weight. The iterative, weight adjustment in the tap weight adjustment process of the Normalised Least mean Square algorithm is

$$
w(n+1)=w(n)+\frac{\mu}{\left\|x(n)^{2}\right\|} x(n) e(n)(3)
$$

Where $\mu$ is the step size controlling the convergence rate, stability and misadjustment and its value should be chosen in the range $0<\mu<2$ to ensure stable Operation. The normalization term in (3) is the Euclidean Norm².

The adaptive step size is $\mu$ is a scalar, and $\delta$ is a small positive number which controls both the convergence rate and steady state which a higher value results in a faster rate of convergence and less stable Drawback:

When the convergence factor $\mu$ is very large the algorithm experiences a gradient noise amplification problem.

\subsection{Reference Algorithm 3-Constrained Stability LMS Algorithm:}

The NLMS algorithm may be viewed as the solution to a constrained optimization problem. The problem of interest may be stated as follows: given the tap-input vector $x(n)$ and the desired response $d(n)$, determine the tap weight vector $\omega(n+1)$ so as to minimize the squared Euclidean norm of the change $\delta \omega(n+1)=\omega(n+1)-\omega(n)$ in the tap-weight vector $\omega(n+1)$ with respect to its old value of the tap weight, $\omega(n)$, subject to the constraint $w^{H}(n+1) x(n)=d(n)$, where $H$ denotes the Hermitian transpose. This constraint means that the a posteriori error sequence vanishes $\left[e^{[k+1]}=d(n)-w^{H}(k+1) x(n)=0\right.$, fork $=$ $n$ ] In order to solve this optimization problem, the method of Lagrange multipliers is used with the Lagrangian function $L\left(w(n+1)=\|\delta w(n+1)\|^{2}+\operatorname{Re}\left[\lambda^{*} e^{(n+1)}(n)\right]\right.$ Where $\lambda^{*}$ is the Lagrange multiplier, thus obtaining 
the well-known adaptation rule with the normalized step size given by $\mu=\hat{\mu}\|x(n)\|^{2}$. The latter constraint is overly restrictive in real applications; thus, if we relax it, another interesting solution can be derived. Where $\delta x(n)=x(n)-x(n-1)$ is the difference between two consecutive input vectors. Hence, the step of the algorithm is Finally, the minimum of the Lagrangian function satisfies the following constrained stability update condition (CS-LMS) $\boldsymbol{w}(\boldsymbol{n}+\mathbf{1})=\boldsymbol{w}(\boldsymbol{n})+\frac{\delta x(n) \delta e^{*}(\boldsymbol{n})}{\|\boldsymbol{\delta}(\boldsymbol{n})\|^{2}}(4)$ The weight adaptation rule can be made more robust by introducing a small positive constant $\epsilon$ into the denominator to prevent numerical instabilities in case of a vanishingly small squared norm $\|\delta x(n)\|^{2}$ and by multiplying the weight increment by a constant step size $\mu$ to control the speed of the adaptation. Note that the equilibrium condition enforces the convergence of the algorithm if $\|\delta x(n)\|^{2} \neq 0$

Several learning algorithms, where the learning relies on the concurrent change of processing variables, have been proposed in the past for de correlation, blind source separation, or de convolution applications. Stochastic information gradient (SIG) algorithms maximize (or minimize) the Shannon's entropy of the sequence of errors using an estimator based on an instantaneous value of the probability density function (pdf) and Parzen windowing. In this way, the CS-LMS algorithm can be considered as a generalization of the single sample-based SIG algorithm using variable kernel density estimators. Once the CS-LMS method has been derived, a comparison is established with the NLMS algorithm.

\section{Advantages:}

(1) CS-LMS and NLMS algorithms converge to the optimal Wiener solution $w_{o}$

(2) for any fixed step size $\mu$, the proposed CS-LMS exhibits improvements in excess minimum squared error

(EMSE) and miss adjustment (M) when compared to the NLMS algorithm.

Drawbacks: An adaptive filter that is not properly regularized will perform very poorly. Regularization plays a fundamental role in adaptive filtering. There are, very likely, many different ways to regularize an adaptive filter. In this paper, we propose one possible way to do it based on a condition that intuitively makes sense. From this condition, we show how to regularize important algorithms: the normalized least-mean-square (NLMS), and show how this algorithm is better than the Constrained Stability LMS algorithm. Later we will apply regularization to the constrained stability LMS algorithm to improve the performance to the maximum extent of the algorithm.

\subsection{Proposed Algorithm-Regularization of the NLMS Algorithm}

The NLMS algorithm is summarized by the following two expressions:

$$
\begin{array}{ll}
=d(n)-\widetilde{y}(n & e(n)=d(n)-x^{T}(n) \widetilde{h}(n) \\
h(n)=h(n-1)+\alpha \frac{e(n) x(n)}{\delta+x^{T}(n) x(n)} &
\end{array}
$$

Where $\alpha(0<\alpha<2)$ is the normalized step-size parameter and $\delta$ is the regularization parameter of the NLMS The question now is how to find $\delta$ ? Since $\boldsymbol{e}(\boldsymbol{n})=\boldsymbol{d}(\boldsymbol{n})-\boldsymbol{x}^{T}(\boldsymbol{n}) \boldsymbol{h}(\boldsymbol{n})$ is the error signal between the desired signal and the estimated signal. We should find $\delta$ in such a way that the expected value of $\mathrm{e}^{2}(\mathrm{n})$ is equal to the variance of the noise,

$$
\text { i.e. }\left[e^{2}(n)\right]=\sigma_{w}^{2}
$$

This is reasonable if we want to attenuate the effects of the noise in the estimator $h(n)$.To derive the optimal according to (11), we assume in the rest that $\mathrm{L} \gg 1$ and $\mathrm{x}(\mathrm{n})$ is stationary, As a result,

$$
\boldsymbol{x}^{T}(\boldsymbol{n}) \boldsymbol{x}(\boldsymbol{n}) \approx \boldsymbol{L} \boldsymbol{\sigma}_{X}^{2}(8)
$$

Developing (5) and using (6), we easily derive the quadratic equation

$$
\delta^{2}-2 \frac{L \sigma_{x}^{2}}{S N R}-\frac{\left(L \sigma_{x}^{2}\right)^{2}}{S N R}=\mathbf{0}(9)
$$

from which we deduce the obvious solution $\delta=\frac{L(1+\sqrt{1+S N R})}{S N R} \sigma_{x}^{2}$

$\boldsymbol{\beta}_{N L M S}=\frac{L(1+\sqrt{1+S N R})}{S N R}$ is the normalized regularization parameter of the NLMS. We see that $\delta$ depends on three elements: the length of the adaptive filter, the variance $\sigma_{x}^{2}$ of the input signal, and the SNR. In both network and adaptive noise o cancellation, the first two elements $\left(L\right.$ and $\sigma_{x}^{2}$ ) are known, while the SNR is often roughly known or can be estimated. 
III. Simulation Results:

The given figure below shows the performance measures of the various algorithm
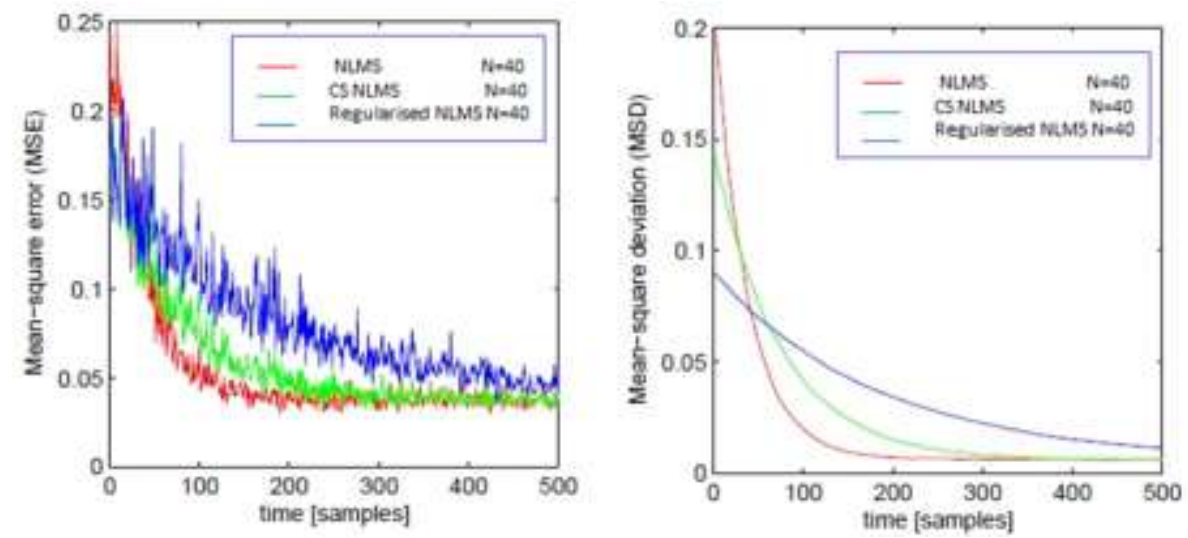

Fig .3 Performance Measure of NLMS,CS NLMS, Regularised NLMS Convergence parameter (a) Mean square value of the error signal (MSE) (b) Mean Square Deviation of the Tap weight vector

The Mean square value of each algorithm is calculated based on the no of iterations and the adaptive algorithms LMS, NLMS, CS-LMS, Regularised NLMS algorithms by considering the tap weight vector order 40 and the $\mu=0.03$ value for the same no of iterations the simulation results are performed. The regularised NLMS performance is best in the Mean square value from the Table.1 it shows better performance considering the less mean square value when compared with constrained stability LMS algorithm the Mean square error value is decreased to a large extent by Regularised NLMS algorithm by regularising the Normalised Least Mean square algorithm.

Table 1.Shows the Mean Square value calculated for the Different Adaptive Algorithms.

The SNR of the various adaptive algorithms Least Mean Square Algorithm, Normalised Least Mean Square Algorithm, Constrained Stability Least mean Square Algorithm and Regularised NLMS algorithm which is having better improvement as shown in the Table.2

\begin{tabular}{|l|c|c|c|c|}
\hline Algorithm & LMS & NLMS & CS-LMS & Regularised NLMS \\
\hline SNR (dB) & 10.9952 & 15.6946 & 21.3696 & 22.213 \\
\hline
\end{tabular}

Table .2 Shows the SNR of the various Adaptive Algorithms

\section{Conclusion}

This paper presents a novel NLMS algorithms based on the concept of regularization, which is used to solve the ill-posed problems occurred in adaptive filtering process. And in this Paper, we proposed a simple condition, for the derivation of an optimal regularization parameter.

This paper implements a new regularised NLMS algorithm having advantages over the Constrained Stability LMS Algorithm. The same procedure can also be implemented with the constrained stability LMS algorithm by regularizing the constrained stability by improving the Signal to noise ratio and the improvement in the convergence speed and reduced Mean square error.

The signals described are taken as the input to the adaptive filter and the filter coefficients are updated using the Constrained Stability LMS algorithm. For a particular value of 500 iterations the simulated result is shown in the figure 3 . The simulated results show that the filtered output closely following the desired signal.

From the comparison graph shown in Fig: 3it is observed that the Regularised NLMS algorithm outperforms all the other algorithms in terms of Mean-Square-error As far as computational burden is concerned, its simplicity is noticed when compared with the NLMS algorithm and LMS Algorithm. Such an aspect, together with convergence improvement and less complexity, is definitive in justifying its application. 
The simulation results for de-noising of the noise added speech wave using standardLMS, NLMS, Constrained Stability LMS and Regularized NLMS for different number of iterations are shown infigure 3. Mean square error is taken as the performance criteria for comparison of adaptive algorithms.

\section{References}

[1] S.Haykin, Adaptive Filter Theory, $4^{\text {th }}$ edition. Englewood Cliffs, NJ: Prentice-Hall, 2002

[2] S.Hykin and A.Steinhardt, Eds., Adaptive Radar Detection and Estimation. New York: Wiley, 1992.

[3] S.L.Marple Jr., Digital Spectral Analysis with Applications. Englewood Cliffs, NJ: Prentice-Hall, 1998.

[4] Leodardo S. Resende,Joao Marcos T. Ramano, Maurice G. Bellanger, "Split Wiener Filtering With Application in Adapive Systems", IEEE Trans.Signal Processing, vol.52, No.3,March 2004.

[5] F. Beaufays, "Transform-domain adaptive filters: An analytical approach," IEEE Trans. Signal Processing, vol. 43, pp. 422-431 Feb. 1995.

[6] J.M.Gorriz, Javier Ramirez, S.Cruces-Alvarez, Carlos G. Puntonet, ElmarW.Lang , and DenizErdogmus, Senor Member IEEE," A Novel LMS Algorithm Applied To Adaptivel Noise Cancellation", IEEE Signal Processing Letters, Vol.16,No.1, pp.34-37, january 2009

[7] ALI H.SAYED, Adaptive Filters, John Willey \& sons, 2003

[8] Paulo Sergio. Ramirez Diniz, Adaptive Filtering Algorithms and Practical Implementations

[9] SimonHaykin , B.Widrow, LeastMeanSquare Adaptive Filters, Johnwilley\& sons, 2003

[10] AlexonderD.Poularikas, ZayedM.Ramadan, Adaptive Filtering Primer With MATLAB, Taylor \& Francis Publishers, 2006.

[11] AllamMousa, MarwaQuados, Sherin Bader, "Adaptive Noise Cancellation Algorithms Sensitivity toParameters", IEEE Conference Publications on ICMCS (Multimedia And Computing Systems) pp. 1-5, 2011.

[12] Md Zia Ur Rahman," Non Stationary Noise Cancellation in Speech Signals Using Efficient Variable Step Size Higher order Filter", IJRRC, vol.2,No.2, pp. 414- 422, April 2011

[13] GeorgiIliev and Nikola Kasabov, "Adaptive Filtering with Averaging in Noise Cancellation forVoice and Speech Recognition", Department of Information Science, University of Otago.

[14] N.Sonbolestan, S.A.hadei,"A Fast Affine Projection Algorith Based on Matching Pursuit in Adaptive noise Cancellation Speech Enhancement",IEEE Conference Publications on ISMS 27-29 jan 2010.

[15] Thamer M. Jamel, HaiderAbd Al-Latifmohamed, "Performance Improvements of Adaptive noise Canceller using New Adajusted Step Size LMS Algorithm", 3rd IEEE Conference on ICSPS august 27282011

[16] E.CIfeachor and B.W.Jervis, Digital Signal Processing, A Practical Approach, Prentice Hall, 2002.

[17] Raymond H. Kwong and Edward W. Johnston, “A Variable Step Size LMS Algorithm”, IEEE Trans. On Signal Processing vol. 40, No.7, pp. 1633-1642, July, 1992

[18] Shoji Makino, Member, IEEE, Yutaka Kaneda, Member, IEEE and Nobuo Koizumi, "Exponentially weighted step size NLMS adaptive filter based on the statistics of a room impulse response", IEEE Trans. on speech and audioProcessing, vol. 1, No.1, pp.101-108, Jan 1993.leads to sub band adaptivealgorithm 